\title{
Duplication of the common carotid artery and the ipsilateral vertebral artery with a fenestration of the contralateral common carotid artery
}

Sir,

Fenestration and duplication of the cervical arteries are considered to be extremely rare anatomic variants. Difficulties can arise when it comes to diagnosing the pathological significance of anomalies of the cervical arteries using imaging procedures. It is important to distinguish between congenital anatomic variants such as duplication and fenestration and acquired anomalies such as dissections, aneurysms and pseudofenestration. ${ }^{[1-3]}$ While the latter implicate a specific and partially extensive therapy, duplications are coincidental findings with no pathological significance.

In a 61-year-old female patient suffering from unexplained dizziness we incidentally observed extremely rare anatomic variants of the cervical arteries. The cervical magnetic resonance (MR) angiography revealed an asymptomatic duplication of the right common carotid artery (CCA) as well as an extracranial duplication of the ipsilateral vertebral artery [Figure 1]. Furthermore, a fenestration of the contralateral CCA was found [Figures 2A, B]. 


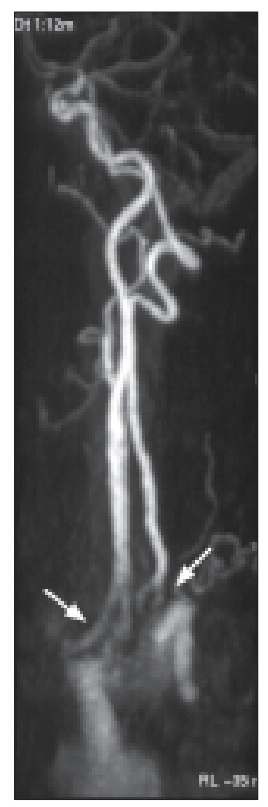

Figure 1: MIP - reconstruction of a TOF - MR - angiography of the right cervical arteries. Duplication of the right common carotid artery (left arrow) and the ipsilateral vertebral artery (right arrow). Both arteries

have two origins from the corresponding subclavian artery.

The duplicated vessels join together to one normal main trunk

In describing anomalies of cervical arteries, the terms duplication and fenestration are to be distinguished clearly, even though they are incorrectly used as synonyms. ${ }^{[1]}$ Arterial duplication is defined as the persistence of a doubly constructed arterial vessel, originally formed in the embryonic phase. This vessel has two separate origins and converges to form one main trunk.

In the case of a duplicate vertebral artery, there are in general two outlets from the subclavian artery which then reunite to a single vessel. A faulty degeneration of the primitive dorsal aorta and two intersegmental arteries is considered to be responsible for the development of duplicate vertebral arteries. ${ }^{[3]}$ The common carotid artery is formed in the fourth and fifth week of embryonic development from parts of the ventral aorta. Disturbances in this period are therefore considered to constitute the trigger for duplication. ${ }^{[4]}$

Duplications are generally rare and, as in the case of our female patient, for the most part incidentally discovered in the course of a cervical cranial MR angiography, a digital subtraction angiography (DSA), computed tomography-angiography (CTA) or an autopsy. On account of their normal wall structure, artery duplications are not linked to an increased predisposition to pathological processes in their supply function. ${ }^{[1]}$ Arterial fenestration on the other hand is to be understood as a short arterial segment with two patent lumens, a single vessel origin and one main trunk into which the two lumen converge. Embryonic misdevelopments are also considered to be responsible for the formation of fenestrations.

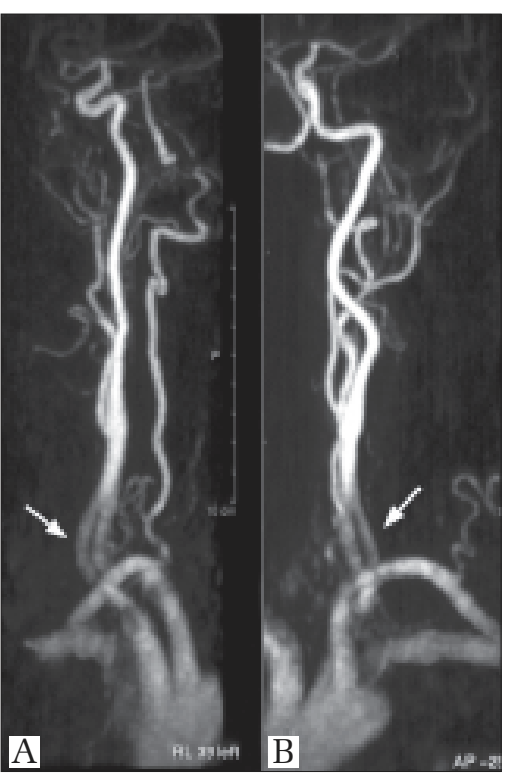

Figure 2A and B: MIP - reconstruction with different projection of a TOF - MR - angiography of the left cervical arteries. Fenestration of the left common carotid artery (arrows) in the middle part between origin from the aortic arch and the bifurcation of the common carotid artery

Fenestrations are of significance in so far as histopathological examinations frequently reveal irregularities in the wall structure of the vessels, in particular in connection with the tunica media. ${ }^{[1]}$ These irregularities could increase the risk of subsequent problems in those areas supplied by the respective arteries (thromboses, embolisms) or be accompanied by other vessel pathologies (aneurysms). ${ }^{[4]}$

In the context of neuroradiological diagnosis, knowledge concerning potential congenital anatomic anomalies in vessels supplying the brain is essential, in order that these may be distinguished from acquired pathological arterial changes such as aneurysms, dissections and pseudofenestrations. ${ }^{[2]}$

In the case described above, incorrectly diagnosing a dissection would have led to therapeutic treatment in the form of, for example, anticoagulation.

Simon Harnier, Andreas Harzheim ${ }^{1}$, Volker Limmroth, Reinhold Horz ${ }^{2}$, Jens Kuhn ${ }^{3}$ Departments of Neurology, ${ }^{1}$ Radiology and ${ }^{2}$ Internal Medicine II, Cologne City Hospitals (Merheim), University of Cologne, Ostmerheimerstrasse 200, 51109, 3'Department of Psychiatry and Psychotherapy, University of Cologne, Kerpener Strasse 62, 50924, Cologne, Germany. E-mail: harniers@kliniken-koeln.de

\section{REFERENCES}

6. Ionete C, Omojola MF. MR angiographic demonstration of bilateral duplication of the extracranial vertebral artery: Unusual course and review of the literature. A.JNR Am J Neuroradiol 2006;27:1304-6.

7. Gailloud P, Carpenter J, Heck DV, Murphy KJ. Pseudofenestration of the cervical internal carotid artery: A pathologic process that simulates an anatomic variant. AJNR Am J Neuroradiol 2004;25:421-4.

8. Gaskill SJ, Heinz ER, Kandt R, Oakes WJ. Bilateral congenital 
anomalies of the extracranial vertebral artery: Management with balloon therapy. Pediatr Neurosurg 1996;25:147-50.

9. Chess MA, Barsotti JB, Chang JK, Ketonen LM, Westesson PL. Duplication of the extracranial internal carotid artery. AJNR Am J Neuroradiol 1995; 16:1545-7.

Accepted Date: 04-06-2008 